\title{
Representation of Spatial Information in Key Areas of the Descending Pain Modulatory System
}

\author{
Christoph Ritter, ${ }^{1}$ Martin N. Hebart, ${ }^{2}$ Thomas Wolbers, ${ }^{3}$ and Ulrike Bingel ${ }^{1}$ \\ ${ }^{1}$ Department of Neurology, University Hospital Essen, University Duisburg-Essen, 45147 Essen, Germany, and ${ }^{2}$ Department of Systems Neuroscience, \\ University Medical Center Hamburg-Eppendorf, 20246 Hamburg, Germany; and ${ }^{3}$ German Center for Neurodegenerative Diseases, 39120 Magdeburg, \\ Germany
}

Behavioral studies have demonstrated that descending pain modulation can be spatially specific, as is evident in placebo analgesia, which can be limited to the location at which pain relief is expected. This suggests that higher-order cortical structures of the descending pain modulatory system carry spatial information about the site of stimulation. Here, we used functional magnetic resonance imaging and multivariate pattern analysis in 15 healthy human volunteers to test whether spatial information of painful stimuli is represented in areas of the descending pain modulatory system. We show that the site of nociceptive stimulation (arm or leg) can be successfully decoded from local patterns of brain activity during the anticipation and receipt of painful stimulation in the rostral anterior cingulate cortex, the dorsolateral prefrontal cortices, and the contralateral parietal operculum. These results demonstrate that information regarding the site of nociceptive stimulation is represented in these brain regions. Attempts to predict arm and leg stimulation from the periaqueductal gray, control regions (e.g., white matter) or the control time interval in the intertrial phase did not allow for classifications above chance level. This finding represents an important conceptual advance in the understanding of endogenous pain control mechanisms by bridging the gap between previous behavioral and neuroimaging studies, suggesting a spatial specificity of endogenous pain control.

\section{Introduction}

The descending pain modulatory system is dedicated to modulating pain selectively by either inhibiting or facilitating nociceptive processing (Millan, 2002; Fields et al., 2006). It comprises cortical areas such as the rostral anterior cingulate cortex (rACC), the dorsolateral prefrontal cortex (dlPFC), and subcortical regions such as the periaqueductal gray (PAG) and is connected to the dorsal horn of the spinal cord, where it can modulate nociceptive information processing at an early stage (Eippert et al., 2009). Endogenous opioids are a key neurotransmitter in this system (Fields et al., 2006). There is ample evidence from clinical and experimental studies that the descending pain modulatory system accounts for interindividual and contextual variations of the pain experience and the susceptibility to chronic pain (Tracey and Mantyh, 2007). A prime example of contextual pain modulation is placebo analgesia, which has been shown to depend on individual functional and structural properties of this network.

Behavioral studies have demonstrated that descending pain modulation can be spatially specific. Placebo analgesia, for

\footnotetext{
Received 0ct. 10, 2013; revised Jan. 30, 2014; accepted Feb. 5, 2014.

Author contributions: C.R., M.N.H., T.W., and U.B. designed research; C.R. performed research; C.R. and M.N.H. analyzed data; C.R. and U.B. wrote the paper.

This work was supported by the German Research Foundation (FOR-1328; B1 789/2-1) and the Federal Ministry of Education and Research (Grant 01G00808).

The authors declare no competing financial interests.

Correspondence should be addressed to Christoph Ritter, Hufelandstrasse 55, 45147 Essen, Germany. E-mail: c.ritter@uke.de.

DOI:10.1523/JNEUROSCI.4342-13.2014

Copyright $\odot 2014$ the authors $\quad 0270-6474 / 14 / 344634-06 \$ 15.00 / 0$
}

example, only occurs at the location at which pain relief is expected (Montgomery and Kirsch, 1996). It has been suggested that this localized effect is subserved by a site-specific action of endogenous opioids (Benedetti et al., 1999). Spinal functional magnetic resonance imaging (fMRI) has revealed a very focal modulation within the spinal dorsal horn corresponding to the site of placebo analgesia (Eippert et al., 2009), which is consistent with the somatotopic organization of the spinal cord, as shown recently in humans using fMRI techniques (Nash et al., 2013). Given that both behavioral and top-down modulatory effects in the spinal cord of descending pain modulatory control can be spatially specific, it is reasonable to assume that spatial information about pain (and its modulation) is represented in higher-order cortical structures of the descending pain modulatory system.

To date, a somatotopic representation for nociceptive information in structures of the descending pain modulatory system has not been reported in functional imaging studies of pain and its modulation in humans. This might partly be due to a lack of sensitivity of mass-univariate approaches computing voxelwise statistics that have been used in most studies of pain, especially if spatial information about pain is not represented in adjacent voxels (as in the classical somatotopy found in motor and somatosensory areas; Penfield and Boldrey, 1937).

The emerging field of multivariate approaches such as multivoxel pattern analysis (MVPA) might help in overcoming these limitations by detecting information that is represented in a distributed fashion to discriminate cognitive or sensory states (Haynes and Rees, 2006; Norman et al., 2006). Here, we used MVPA in combination with a robust heat pain paradigm applied 


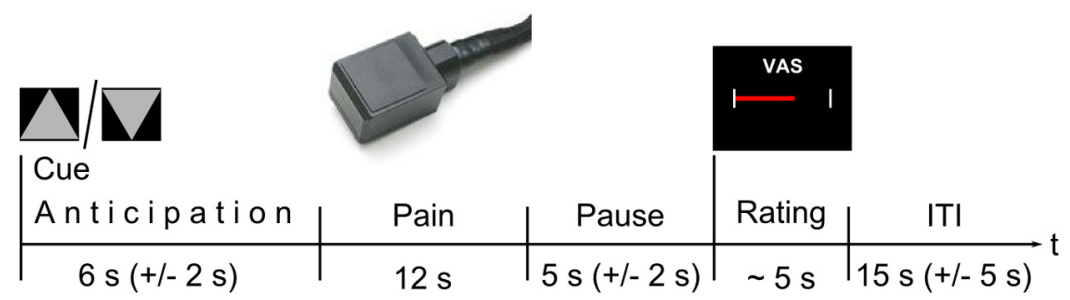

Figure 1. Experimental paradigm. Trial structure during imaging. Triangles pointing either upward or downward signal the next painful stimulus. The cue remains visible until the VAS is presented. After completion of the rating, a variable ITI follows, during which time a white crosshair is shown until the next cue appears.

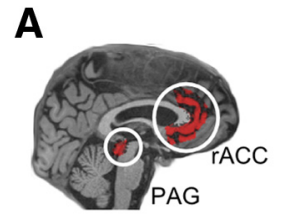

B

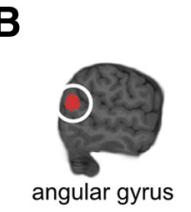

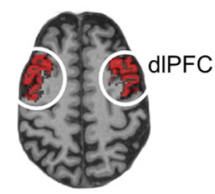
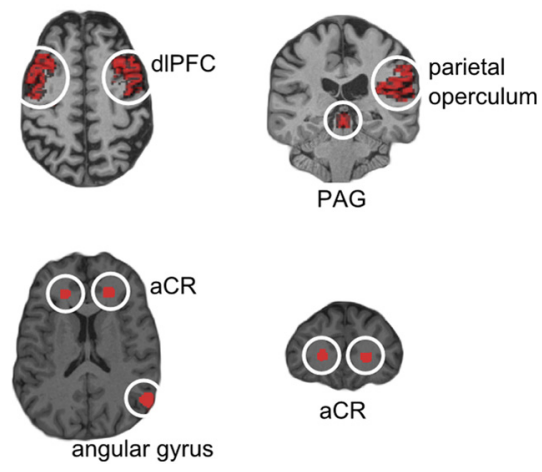

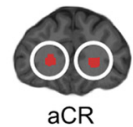

$\mathrm{aCR}$
Figure 2. ROls shown on an individual brain. $\boldsymbol{A}$, Areas involved in nociception: PAG, rACC, dIPFC, parietal operculum. $B$, Control regions: angular gyrus, aCR.

to the arm and leg of healthy volunteers to test whether somatotopic information of painful stimuli is represented in areas of the descending pain modulatory system.

\section{Materials and Methods}

Subjects. Fifteen right-handed healthy volunteers (3 females; mean age 24.6 years, range $19-32$ years) took part in the study. All subjects had normal heat pain thresholds at the sites of stimulation (Rolke et al., 2006) and had no known history of neurological or psychiatric diseases, including recurrent or chronic pain. The study was conducted in accordance with the Declaration of Helsinki and was approved by the local ethics committee. All participants gave written informed consent to participate and were free to withdraw from the study at any time.

Experimental paradigm. The experiment comprised an introductory session and an imaging session performed on the same day.

Introductory session. During the introductory session, participants were familiarized with the heat pain stimuli and the rating procedure and individual temperature levels used during the experiment were determined. First, the individual heat pain thresholds at the two sites of stimulus application (i.e., left inner forearm and lateral calf) were determined using the Method of Limits (Fruhstorfer et al., 1976). Thresholds were obtained using ramped stimuli $\left(1^{\circ} \mathrm{C} / \mathrm{s}\right.$ increase in temperature, starting at a baseline of $35^{\circ} \mathrm{C}$ and with an upper limit of $50^{\circ} \mathrm{C}$ to avoid tissue damage). Subjects indicated their first painful sensation by pressing a button. To familiarize the participant with the rating on the Visual Analog Scale (VAS end points: $0=$ no pain, $100=$ unbearable pain), which was presented on the computer screen in front of the participants, $512 \mathrm{~s}$ stimuli with a temperature $\sim 0.5^{\circ} \mathrm{C}$ above the individual pain threshold were applied to the volar forearm. Subsequently, a calibration procedure was performed to identify the temperature level at which the stimulation was rated as VAS 60. To this end, subjects were presented with stimuli of varying temperature levels around their individual pain threshold (range: $-1^{\circ} \mathrm{C}<$ pain threshold $<+3.5^{\circ} \mathrm{C}$, in increments of $0.5^{\circ} \mathrm{C}$; stimulus duration: $12 \mathrm{~s}$; each temperature was applied twice) and rated the intensity of each stimulus on the VAS. A linear regression analysis was used to calculate the temperature that corresponded to VAS 60. Painful the volunteers. stimuli were delivered using two MRcompatible thermal devices (TSA-II and Pathway (ATS part); Medoc), which were identical with respect to their relevant features (e.g., contact area $3 \times 3 \mathrm{~cm}$, identical capabilities regarding temperature rise and fall times, etc.). The software package Presentation was used for stimulus delivery and logging of button presses (version 14.9, build 07.19.11; Neurobehavioral Systems).

Imaging session. After positioning the volunteer in the MR scanner, a short recalibration of the stimulation intensities with four stimuli on each limb was performed to check whether the temperature levels determined at the calibration session evoked a pain sensation of VAS 60. The target temperature was adjusted if necessary. During the actual experiment, a total of 80 pain stimuli (40 on each limb) were applied. Painful stimuli were presented in blocks of five consecutive trials applied to the same stimulation site. To allow for the investigation of anticipatory responses in addition to stimulus-related responses, a visual cue informed the participants about the site (i.e., arm/leg) at which the next stimulus would be applied. Imaging was performed in two equal consecutive sessions (40 painful stimuli were applied during each session) to account for possible slow signal drifts of the MR scanner and fatigue of

Each trial consisted of the following phases: anticipation (triangle, $6 \pm$ $2 \mathrm{~s}$ ), pain (triangle still visible, $12 \mathrm{~s}$ ), pause (triangle still visible, $5 \pm 2 \mathrm{~s}$ ), rating (VAS, $\sim 5 \mathrm{~s}$ ), and intertrial interval (ITI; white crosshair, $15 \pm 5 \mathrm{~s}$ ), resulting in a total duration of $43 \mathrm{~s}$ (Fig. 1). A gray triangle pointing upward signaled a subsequently applied stimulation of the arm. A downward pointing triangle signaled the stimulation of the leg. Subjects were instructed to press a button at the beginning of each anticipation phase to ensure that their attention was directed to the upcoming stimulation. The triangle was shown throughout the whole trial until the display switched to the VAS for the intensity rating of the preceding stimulus. The stimulation order (i.e., stimulation applied to the arm or leg) was pseudorandomized to ensure that stimulation site changed after five consecutive trials.

Data acquisition. Imaging was performed using a $3 \mathrm{~T}$ MR scanner (TimTrio; Siemens) and a 32-channel head coil. The functional images were acquired with a $\mathrm{T}^{*}$ weighted gradient-echo echo-planar-imaging sequence $(\mathrm{TR}=1970 \mathrm{~ms}$, TE $=26 \mathrm{~ms}, 2 \times 2 \times 2 \mathrm{~mm}$ voxel size, parallel imaging, 33 slices, 25\% gap, FOV $208 \mathrm{~mm}$, descending) covering an area between at least the brainstem and the secondary somatosensory cortices, which were determined on scout scans before scanning the actual experiment. Subsequently, a structural T1-weighted magnetization-prepared rapid acquisition gradient echo sequence was performed (slice thickness, $1 \mathrm{~mm}$; TR, $2.30 \mathrm{~s}$; TE, $2.98 \mathrm{~ms}$; flip angle, $9^{\circ}$; FOV, $256 \times 256 \mathrm{~mm}$ ) that covered the whole brain. Note that the two experimental conditions were equally represented in both imaging sessions.

Image processing. All analyses were performed in the individual anatomical space. The functional images were preprocessed using SPM8 (www.fil.ion.ucl.ac.uk/spm/). The first six images were discarded to allow for T1 saturation. The remaining images were corrected for slice acquisition timing, then realigned to the first volume and unwarped to account for interactions of head motion with inhomogeneities of the magnetic field. Finally, all images were smoothed with a $4 \mathrm{~mm}$ Gaussian kernel to improve the signal-to-noise-ratio for decoding (Op de Beeck, 2010; Schwarzkopf et al., 2011). The structural images were segmented and bias corrected using SPM8's segment function to obtain the parameters for the inverse normalization of the region of interest (ROI) masks and a gray matter (GM) image that was used to restrict the masks to gray matter voxels. The segmented GM image was thresholded at 50 (percentage of robust range) and binarized.

Analysis was restricted to ROIs that included core regions of the descending pain modulatory control system observed during placebo analgesia, the PAG, dlPFC, and rACC. The parietal operculum served as a "positive" control with a known somatotopic representation of nocicep- 
A

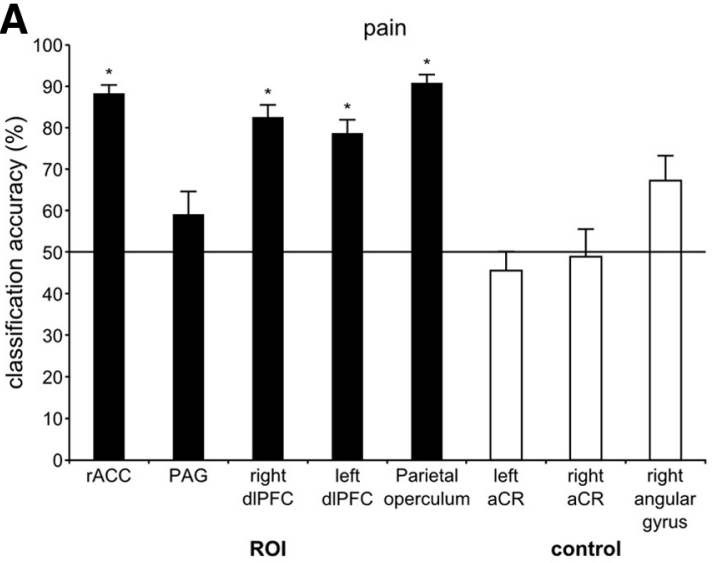

C

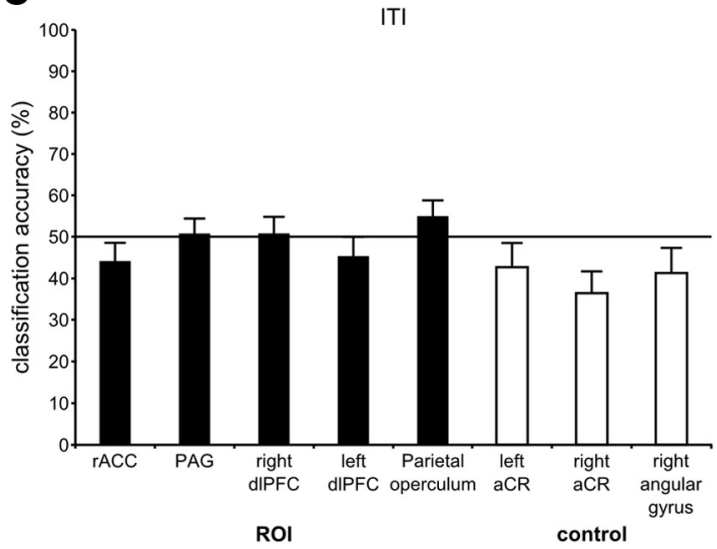

B

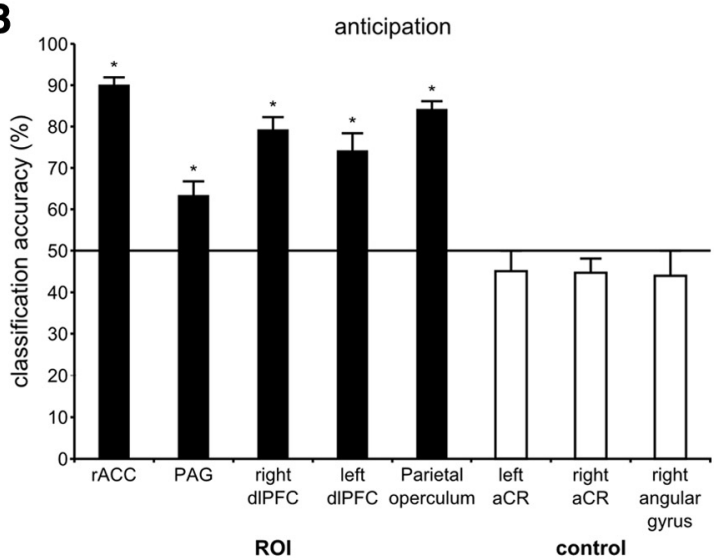

D

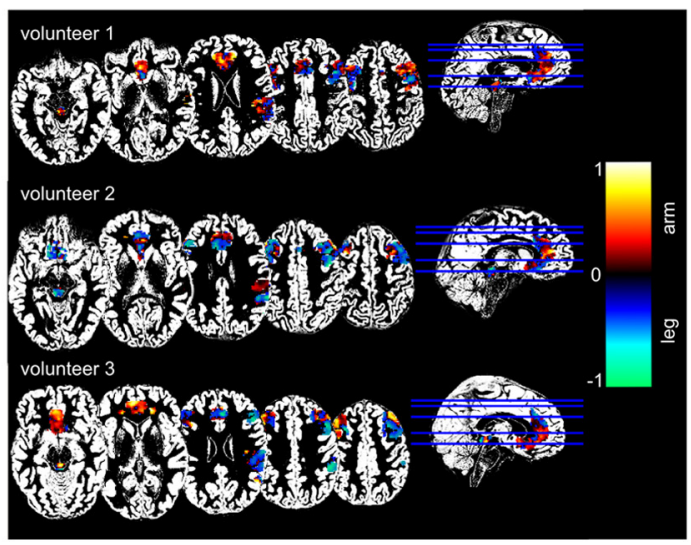

Figure 3. Results. $\boldsymbol{A}$, Classification accuracies in ROIs (rACC, PAG, left and right dIPFCS, parietal operculum) and control ROls (aCR and right angular gyrus) during painful stimulation. $\boldsymbol{B}$, Classification accuracies in ROIs and control ROIs during anticipation of the painful stimulus. C, Classification accuracies in ROIs and control ROIs during the ITI. Error bars indicate the SEM. ${ }^{*} p$-value $<$ 0.003 accounting for multiple comparisons using Bonferroni correction. D, Reconstructed activity patterns of three representative volunteers based on the methodology of Haufe et al. (2014). Strength of class-related information. The hot color scheme depicts a stronger relationship with stimulation of the arm, whereas the winter color scheme depicts a stronger relationship with stimulation of the leg (in arbitrary units).

tive information for the studied body parts (Bingel et al., 2004). "Negative" control regions where no somatotopic representation was expected comprised the anterior corona radiata (bilateral) and the right angular gyrus. All ROIs were derived from the Harvard-Oxford Atlas, which is part of the FMRIB software library (FSL; http://www.fmrib.ox.ac. $\mathrm{uk} / \mathrm{fs} \mathrm{l} /$ ), using fslroi and fslmaths command line tools to threshold and binarise images. The rACC, dlPFC, and PAG masks were constructed according to Stein et al. (2012); in addition, the rACC mask was cut at $y<$ $30 \mathrm{~mm}$ at the genu of the corpus callosum, which approximately marks a cytoarchitectonically defined border between affective and cognitive divisions of the ACC (Bush et al., 2000). All masks were transformed into the individual anatomical space using the normalize function of SPM8 and the parameters obtained from segmenting the individual T1weighted structural image. The resulting mask images were multiplied with the thresholded, binarized, and segmented high-resolution GM image. Finally, the masks were coregistered with the mean EPI image and resliced (Fig. 2).

Pattern classification. Pattern classification was performed using the Princeton MVPA toolbox (http://code.google.com/p/princeton-mvpatoolbox/) and a linear support vector machine (LIBSVM; Chang and Lin, 2011) with optimized soft margin parameter $c$. This optimization was done by applying a nested cross-validation scheme, which means that during cross-validation, an additional level of cross-validation was applied to the training dataset to determine the optimal parameter for $c$. That nested cross-validation approach enabled us to refrain from using the test data to optimize the regularization parameter $c$ and thus circumvented the problem of circular analysis (Brodersen et al., 2012).

Pattern classification was separately applied to data from three different time periods: the anticipation period, stimulation period, and the ITI.
Time courses were extracted from the preprocessed images (see Image processing, above) and stored in a $2 \mathrm{D}$ matrix (nVoxels $\times$ nTRs), discarding $3 \mathrm{D}$ information for the sake of memory efficiency and facilitation of data processing. The data were high-pass filtered (cutoff: $128 \mathrm{~s}$ ), $z$-transformed, and the regressors that determined the onsets of the trials were shifted by 3 TRs to account for hemodynamic delay (Kamitani and Tong, 2006). TRs were averaged across each of the time periods (e.g., 6 TRs for the stimulation period) except the ITI, for which the first TR was discarded to prevent a "carry-over" of the stimulation period. Trials were then averaged according to block length (five trials) to improve the signal-to-noise ratio of the BOLD signal estimates entering the classifier, yielding eight samples per category. Finally, a leave-one-out crossvalidation scheme was applied. Data were divided into training and test sets, training the classifier on 15 subsets and testing on the one withheld. This test was repeated 16 times, with each different block serving as a test subset once (Pereira et al., 2009). This resulted in a classification accuracy ranging between 0 and $100 \%$ that quantified how accurately the classifier was able to distinguish between stimulation of the arm and leg. These procedures were performed separately for each ROI and each control region per subject. Finally, a mean accuracy was computed for the whole group and the accuracies of these regions were tested against chance level (i.e., 50\%) using one-tailed $t$ tests. Bonferroni correction was applied for multiple comparisons, so that $p$-values $<0.003$ were considered significant. Moreover, we also conducted a repeated-measures ANOVA on the classification accuracies with factors time period (three levels: anticipation, stimulation, and ITI) and ROI (eight levels: rACC, PAG, both dlPFCs, parietal operculum, both aCRs, and right angular gyrus). Post hoc tests were performed when legitimate. All behavioral data and accuracy data were analyzed using SPSS 13. 
Accounting for ROI size. To account for any potential bias induced by the different sizes of our ROIs, we corrected our classification estimation for ROI size using a bootstrapping approach as used in Haushofer et al. (2008). We matched the size of each ROI (rACC, dlPFC, PAG, and parietal operculum) to the size of our smallest gray matter control ROI in the angular gyrus ROI by randomly selecting $92( \pm 28 \mathrm{SD})$ voxels out of our ROIs for classification. This selection process was repeated 1000 times and results of these bootstrap samples were averaged to determine the mean classification accuracy unbiased by ROI size. All remaining analysis steps were the same as described Pattern classification, above. This approach assured that the higher classification accuracies found in our ROIs were not confounded by their sizes.

Reconstruction of patterns. We applied the approach suggested by Haufe et al. (2014) to reconstruct the activity patterns. In short, we applied the following algorithm:

$$
A=\operatorname{cov}(X)^{\star} W^{\star} \operatorname{cov}(S)^{-1}
$$

where $A$ is the reconstructed pattern, $W$ is the weight vector, $\operatorname{cov}(X)$ is the $n$-by- $p$ covariance matrix of the data (with $n$ voxels and $p$ samples), and $\operatorname{cov}(S)$ is the source covariance, defined as $W^{\mathrm{T} \star} X$. Please note that the reconstructed patterns depend on the (limited) data and the classifier used and may be different for other classifiers or different parameter values (Fig. 3D).

\section{Results}

\section{Behavioral data}

The mean heat pain threshold $( \pm \mathrm{SD})$ was $44.36 \pm 2.82^{\circ} \mathrm{C}$ on the arm and $45.14 \pm 3.09^{\circ} \mathrm{C}$ on the leg, which is within in the normal range for healthy volunteers of this age range (Rolke et al., 2006). A paired two-tailed $t$ test revealed no significant difference $\left(t_{(14)}=-1.074\right.$, n.s.). The mean temperature $( \pm S D)$ used to induce a VAS of 60 was $46.43 \pm 1.02^{\circ} \mathrm{C}$ on the arm and $46.69 \pm 1.3^{\circ} \mathrm{C}$ on the leg, with no significant difference between stimulation sites $\left(t_{(14)}=-0.873\right.$, n.s.; paired two-tailed $t$ test). The mean pain ratings ( \pm SD) were $52.85 \pm$ 11.14 for painful stimulation of the arm and $48.65 \pm 13.51$ for the leg. A paired two-tailed $t$ test comparing ratings for arm and leg yielded no significant result $\left(t_{(14)}=0.994\right.$, n.s. $)$. The number of button presses and reaction times did not differ significantly between conditions.

\section{Univariate analysis of imaging data}

A classical univariate GLM analysis testing for differences between the two conditions (contrasts: arm $>$ leg and leg $>$ arm) did not reveal any significant differences in our ROIs at a threshold of $p<0.001$ (uncorrected for multiple comparisons).

\section{Classification accuracies}

Because this is a two-class problem (arm vs leg), the chance level is $50 \%$ and perfect classification would have been $100 \%$. During painful stimulation, we achieved significant classification accuracies in the rACC, both dlPFCs, and the contralateral parietal operculum. Classification accuracy in the PAG did not exceed chance level (Fig. $3 A$, Table 1). In the control regions (left and right anterior corona radiata, right angular gyrus), classification accuracies were not significantly different from chance level, demonstrating the specificity of our results to the regions involved in pain modulation. The bootstrapping analysis for the stimulation phase accounting for ROI size confirmed these results and revealed significant classification accuracies in the rACC, both dlPFCs, and the contralateral parietal operculum (Table 1). Analysis of the anticipation phase yielded significant classification accuracies in all ROIs (Fig. 3B, Table 1). In contrast, analyses of the
Table 1. Classification accuracies and statistics in ROIs and control regions during painful stimulation, anticipation, and ITI

\begin{tabular}{llllc}
\hline ROI & Accuracy ( \pm SD) & df & $t$ & $p$ \\
\hline Painful stimulation & & & & \\
rACC & $88 \pm 9 \%$ & 14 & 17.5 & $<0.001^{*}$ \\
PAG & $59 \pm 22 \%$ & 14 & 1.6 & 0.07 \\
Left dIPFC & $79 \pm 13 \%$ & 14 & 8.5 & $<0.001^{*}$ \\
Right dIPFC & $83 \pm 12 \%$ & 14 & 10.2 & $<0.001^{*}$ \\
Parietal operculum & $91 \pm 9 \%$ & 14 & 18.7 & $<0.001^{*}$ \\
Left aCR & $46 \pm 17 \%$ & 14 & -0.9 & 0.82 \\
Right aCR & $49 \pm 25 \%$ & 14 & -0.1 & 0.55 \\
Right angular gyrus & $65 \pm 19 \%$ & 14 & 3.0 & 0.004 \\
Painful stimulation (bootstrapping) & $78 \pm 12 \%$ & 14 & 8.6 & $<0.001^{*}$ \\
rACC & $54 \pm 24 \%$ & 14 & 0.7 & 0.25 \\
PAG & $67 \pm 15 \%$ & 14 & 4.3 & $<0.001^{*}$ \\
Left dIPFC & $70 \pm 20 \%$ & 14 & 3.9 & $<0.001^{*}$ \\
Right dIPFC & $84 \pm 13 \%$ & 14 & 10.0 & $<0.001^{*}$ \\
Parietal operculum & & & & \\
Anticipation & $90 \pm 7 \%$ & 14 & 21.0 & $<0.001^{*}$ \\
rACC & $63 \pm 14 \%$ & 14 & 3.8 & $<0.001^{*}$ \\
PAG & $74 \pm 17 \%$ & 14 & 5.7 & $<0.001^{*}$ \\
Left dIPFC & $79 \pm 12 \%$ & 14 & 9.1 & $<0.001^{*}$ \\
Right dIPFC & $84 \pm 8 \%$ & 14 & 17.0 & $<0.001^{*}$ \\
Parietal operculum & $45 \pm 18 \%$ & 14 & -1.0 & 0.83 \\
Left aCR & $45 \pm 13 \%$ & 14 & -1.5 & 0.92 \\
Right aCR & $44 \pm 22 \%$ & 14 & -1.0 & 0.84 \\
Right angular gyrus & & & & \\
ITI & $44 \pm 18 \%$ & 14 & -1.3 & 0.89 \\
rACC & $51 \pm 15 \%$ & 14 & 0.2 & 0.42 \\
PAG & $45 \pm 19 \%$ & 14 & -1.0 & 0.82 \\
Left dIPFC & $51 \pm 16 \%$ & 14 & 0.2 & 0.42 \\
Right dIPFC & $55 \pm 16 \%$ & 14 & 1.2 & 0.12 \\
Parietal operculum & $43 \pm 23 \%$ & 14 & -1.2 & 0.88 \\
Left aCR & $37 \pm 21 \%$ & 14 & -2.5 & 0.99 \\
Right aCR & $42 \pm 23 \%$ & 14 & -1.4 & 0.91 \\
Right angular gyrus & & & \\
\hline
\end{tabular}

*Significant $p$-value $<0.003$ (accounting for multiple comparisons using Bonferroni correction).

control regions (left and right anterior corona radiata, right angular gyrus) yielded no classification accuracies that were significantly different from chance level. Further, in the ITI, the classification performance did not exceed chance level in the ROIs or control regions (Fig. 3C).

The ANOVA yielded both significant main effects (time period: $F_{(2,28)}=51.054, p<0.001 ;$ ROI: $\left.F_{(7,98)}=35.022, p<0.001\right)$ and a significant time $\times$ ROI interaction $\left(F_{(14,196)}=4.789, p<\right.$ 0.001). Post hoc tests revealed the following results. Classification accuracies in the rACC, bilateral dIPFCs, and parietal operculum were significantly higher compared with each of the control regions during the anticipation and stimulation period. Classification accuracies in the rACC and bilateral dlPFCs were significantly higher during anticipation and stimulation compared with the ITI. There were no differences in classification accuracies for rACC or dlPFC between anticipation and stimulation or between the rACC and both dlPFCs, either in the stimulation or in the anticipation phase $(p<0.00018$, Bonferroni corrected for multiple comparisons).

\section{Discussion}

The descending pain modulatory system is a crucial determinant of acute and chronic pain states and is known to exert localized effects. Here, we investigated whether spatial information of nociceptive stimuli is represented in the rACC, the dlPFC, and the PAG as key areas of this system. We show that the site of nociceptive stimulation (arm or leg) can be successfully decoded from 
brain activity in rACC and dlPFC during the anticipation and stimulation period. This indicates that spatial information regarding the site of nociceptive stimulation is represented in these brain regions. As expected, we also classified activity in the contralateral parietal operculum that was used as a control region because of its known somatotopic representation of nociceptive information (Bingel et al., 2004; Brooks et al., 2005). The PAG, in contrast, did not allow for classifications above chance level. Importantly, no significant effects were found for decoding from white matter (anterior corona radiata left and right) or the right angular gyrus as a gray matter control area. Furthermore, attempts to distinguish between arm and leg stimulation from activity during the ITI yielded no significant effects.

Although the rACC and dlPFC are involved in a variety of cognitive-affective processes, both neuroimaging (for review, see Wiech et al., 2008; Tracey and Dickenson, 2012) and functional lesion studies (Krummenacher et al., 2010) indicate the dlPFC and the rACC as key cortical brain areas that initiate and subsequently mediate top-down modulatory pain control mechanisms. The latter have been shown to modulate spinal nociceptive processing and pain perception in a spatially specific manner. Our findings indicate that information about the site of nociceptive stimulation is represented in these higher cortical areas of top-down pain control, as expected from their functional role in top-down pain control. Intriguingly, spatial information regarding painful stimulation can already be classified from the anticipation period, which is in line with engagement of the descending pain modulatory system already in the anticipation phase of upcoming painful stimulation (Bingel and Tracey, 2008).

To our knowledge, only one fMRI study has previously examined somatotopic effects in the cingulate cortex and found differential activity after both nonpainful and painful electrical stimulation of either the median or tibial nerve (Arienzo et al., 2006), but in areas of the cingulate cortex dorsal to those commonly observed during pain modulation. Our study is the first to demonstrate such a somatotopic effect in key regions of the descending pain modulatory system. Although the PAG is an important downstream control area of the descending pain modulatory system (Bingel and Tracey, 2008; Heinricher et al., 2009) and animal data have previously suggested at least a coarse somatotopic representation of body quadrants (Soper and Melzack, 1982; Kasman and Rosenfeld, 1986), it did not allow for classification in our study. This is likely to be explained by the particularly small size, shape, and location around the aqueduct of the PAG that renders normalization procedures difficult and reduces the sensitivity of MVPA approaches.

The somatotopic representation of nociceptive information observed in our study represents an important conceptual advance because it may explain both the behavioral effects of spatially directed placebo analgesia and the corresponding spatially specific spinal fMRI effects reported previously (Montgomery and Kirsch, 1996; Benedetti et al., 1999; Eippert et al., 2009). The successful decoding of spatial information in our study raises the question about the topography of this information. Given the negative results of univariate neuroimaging approaches, it seems unlikely that spatial information is represented in a classical somatotopical fashion with adjacent areas of the body being mapped to adjacent areas of the brain (Foerster, 1931; Penfield and Boldrey, 1937). Instead, as shown in Figure 3, the topographical representation seems to be highly variable across participants and "fractured" rather than contiguous within individuals.

\section{Link to chronic pain conditions}

Our finding that spatial information of nociceptive information and the resulting pain experience is mirrored in areas of the descending pain modulatory system might open a new avenue in understanding the development of chronic pain conditions. Although pain tends to spread with increasing chronification, many chronic pain conditions in which no actual tissue damage is present are predominantly located in distinct body parts, such as chronic low back pain, chronic headache disorders, or chronic pelvic pain. Given that top-down factors such as descending pain control are an important determinant for the susceptibility and maintenance of chronic pain, a site-specific failure of descending pain control in combination with other contextual factors might explain the evolution of topologically specific pain disorders.

\section{Conclusion}

In sum, our study reveals that spatial information of nociceptive stimulation is represented in cortical key areas of the descending pain modulatory system. Future research will have to further investigate corticosubcortical and spinal interactions in a somatotopically specific manner and elucidate the relevance of this observation for the development of chronic pain states.

\section{References}

Arienzo D, Babiloni C, Ferretti A, Caulo M, Del Gratta C, Tartaro A, Rossini PM, Romani GL (2006) Somatotopy of anterior cingulate cortex (ACC) and supplementary motor area (SMA) for electric stimulation of the median and tibial nerves: an fMRI study. Neuroimage 33:700-705. CrossRef Medline

Benedetti F, Arduino C, Amanzio M (1999) Somatotopic activation of opioid systems by target-directed expectations of analgesia. J Neurosci 19: 3639-3648. Medline

Bingel U, Tracey I (2008) Imaging CNS modulation of pain in humans. Physiology (Bethesda) 23:371-380. CrossRef Medline

Bingel U, Lorenz J, Glauche V, Knab R, Gläscher J, Weiller C, Büchel C (2004) Somatotopic organization of human somatosensory cortices for pain: a single trial fMRI study. Neuroimage 23:224-232. CrossRef Medline

Brodersen KH, Wiech K, Lomakina EI, Lin CS, Buhmann JM, Bingel U, Ploner M, Stephan KE, Tracey I (2012) Decoding the perception of pain from fMRI using multivariate pattern analysis. Neuroimage 63:11621170. CrossRef Medline

Brooks JC, Zambreanu L, Godinez A, Craig AD, Tracey I (2005) Somatotopic organisation of the human insula to painful heat studied with high resolution functional imaging. Neuroimage 27:201-209. CrossRef Medline

Bush G, Luu P, Posner MI (2000) Cognitive and emotional influences in anterior cingulate cortex. Trends Cogn Sci 4:215-222. CrossRef Medline

Chang CC, Lin CJ (2011) LIBSVM: a library for support vector machines. ACM Transactions on Intelligent Systems and Technology 2:27:21-27:27.

Eippert F, Finsterbusch J, Bingel U, Büchel C (2009) Direct evidence for spinal cord involvement in placebo analgesia. Science 326:404. CrossRef Medline

Fields HL, Basbaum AI, Heinricher MM (2006) Central nervous system mechanisms of pain modulation. In: Wall and Melzack's textbook of pain (McMahon SB, Koltzenburg M, eds), pp 125-152. London: Elsevier.

Foerster O (1931) The cerebral cortex in man. Lancet 218:309-312. CrossRef

Fruhstorfer H, Lindblom U, Schmidt WC (1976) Method for quantitative estimation of thermal thresholds in patients. J Neurol Neurosurg Psychiatry 39:1071-1075. CrossRef Medline

Haufe S, Meinecke F, Görgen K, Dähne S, Haynes JD, Blankertz B, Bießmann F (2013) On the interpretation of weight vectors of linear models in multivariate neuroimaging. Neuroimage 87:96-110. CrossRef Medline

Haushofer J, Livingstone MS, Kanwisher N (2008) Multivariate patterns in object-selective cortex dissociate perceptual and physical shape similarity. PLoS Biol 6:e187. CrossRef Medline

Haynes JD, Rees G (2006) Decoding mental states from brain activity in humans. Nat Rev Neurosci 7:523-534. CrossRef Medline 
Heinricher MM, Tavares I, Leith JL, Lumb BM (2009) Descending control of nociception: Specificity, recruitment and plasticity. Brain Res Rev 60: 214-225. CrossRef Medline

Kamitani Y, Tong F (2006) Decoding seen and attended motion directions from activity in the human visual cortex. Curr Biol 16:1096-1102. CrossRef Medline

Kasman GS, Rosenfeld JP (1986) Opiate microinjections into midbrain do not affect the aversiveness of caudal trigeminal stimulation but produce somatotopically organized peripheral hypoalgesia. Brain Res 383:271278. CrossRef Medline

Krummenacher P, Candia V, Folkers G, Schedlowski M, Schönbächler G (2010) Prefrontal cortex modulates placebo analgesia. Pain 148:368374. CrossRef Medline

Millan MJ (2002) Descending control of pain. Prog Neurobiol 66:355-474. CrossRef Medline

Montgomery G, Kirsch I (1996) Mechanisms of placebo pain reduction: an empirical investigation. Psychological Science 7:174-176. CrossRef

Nash P, Wiley K, Brown J, Shinaman R, Ludlow D, Sawyer AM, Glover G, Mackey S (2013) Functional magnetic resonance imaging identifies somatotopic organization of nociception in the human spinal cord. Pain 154:776-781. CrossRef Medline

Norman KA, Polyn SM, Detre GJ, Haxby JV (2006) Beyond mind-reading: multi-voxel pattern analysis of fMRI data. Trends Cogn Sci 10:424-430. CrossRef Medline

Op de Beeck HP (2010) Against hyperacuity in brain reading: spatial smoothing does not hurt multivariate fMRI analyses? Neuroimage 49: 1943-1948. CrossRef Medline

Penfield W, Boldrey E (1937) Somatic motor and sensory representation in the cerebral cortex of man as studied by electrical stimulation. Brain 60:389-443. CrossRef

Pereira F, Mitchell T, Botvinick M (2009) Machine learning classifiers and fMRI: a tutorial overview. Neuroimage 45:S199-S209. CrossRef Medline

Rolke R, Baron R, Maier C, Tölle TR, Treede RD, Beyer A, Binder A, Birbaumer N, Birklein F, Bötefür IC, Braune S, Flor H, Huge V, Klug R, Landwehrmeyer GB, Magerl W, Maihöfner C, Rolko C, Schaub C, Scherens A, Sprenger T, Valet M, Wasserka B (2006) Quantitative sensory testing in the German Research Network on Neuropathic Pain (DFNS): standardized protocol and reference values. Pain 123:231-243. CrossRef Medline

Schwarzkopf DS, Sterzer P, Rees G (2011) Decoding of coherent but not incoherent motion signals in early dorsal visual cortex. Neuroimage 56 : 688-698. CrossRef Medline

Soper WY, Melzack R (1982) Stimulation-produced analgesia: evidence for somatotopic organization in the midbrain. Brain Res 251:301-311. CrossRef Medline

Stein N, Sprenger C, Scholz J, Wiech K, Bingel U (2012) White matter integrity of the descending pain modulatory system is associated with interindividual differences in placebo analgesia. Pain 153:2210-2217. CrossRef Medline

Tracey I, Dickenson A (2012) SnapShot: Pain perception. Cell 148:13081308.e2. CrossRef Medline

Tracey I, Mantyh PW (2007) The cerebral signature for pain perception and its modulation. Neuron 55:377-391. CrossRef Medline

Wiech K, Ploner M, Tracey I (2008) Neurocognitive aspects of pain perception. Trends Cogn Sci 12:306-313. CrossRef Medline 